\title{
A Model for Temporal Dynamics of Brown Rot Spreading in Fruit Orchards
}

\author{
Daniele Bevacqua, ${ }^{\dagger}$ Bènèdicte Quilot-Turion, and Luca Bolzoni
}

First author: UR 1115 Plantes et Systèmes de culture Horticoles, INRA, Avignon, France; second author: UR 1052 Génétique et Amélioration des Fruits et Légumes, INRA, Avignon, France; and third author: Risk Analysis Unit, Istituto Zooprofilattico Sperimentale della Lombardia e dell'Emilia Romagna, Parma, Italy.

Accepted for publication 23 November 2017.

\begin{abstract}
Brown rot, caused by Monilinia spp., is a major disease of stone fruit and, in favorable environmental conditions and in the absence of fungicide treatments, it causes important economic losses. In the present work, we propose a modification of classical susceptible, exposed, infectious and removed compartmental models to grasp the peculiarities of the progression of brown rot epidemics in stone fruit orchards in the last stage of the fruit growth (i.e., from the end of the pit hardening to harvest time). Namely, we took into account (i) the lifespan of airborne spores; (ii) the dependence of the latent period on the cuticle crack surface area, which itself varies in time with fruit growth; (iii) the impossibility of recovery in infectious fruit; and (iv) the abrupt interruption of disease development by the elimination of the host fruit at harvest time. We parametrized the model by using field data

from a peach Prunus persica orchard infected by Monilinia laxa and M. fructicola in Avignon (southern France). The basic reproduction number indicates that the environmental conditions met in the field were extremely favorable to disease development and the model closely fitted the temporal evolution of the fruit abundance in the different epidemiological compartments. The model permits us to highlight crucial mechanisms undergoing brown rot build up and to evaluate the consequences of different agricultural practices on the quantity and quality of the yield. We found that winter sanitation practices (which decrease the initial infection incidence) and the control of the fruit load (which affects the host fruit density and the single fruit growth trajectory) can be effective in controlling brown rot in conjunction with or in place of fungicide treatments.
\end{abstract}

Brown rot caused by Monilinia spp. is considered one of the most serious diseases of fruit. The genus Prunus, which includes species with important economic value such as peach, plum, apricot, cherry, and almond, is affected by M. fructicola and M. laxa and, to a lesser extent, by $M$. fructigena, which can affect also apple and pear (Gibert et al. 2005). Brown rot is present in all temperate regions and is responsible for important economic losses (Oliveira Lino et al. 2016). Today its control is mostly implemented by the use of chemicals with inherent environmental costs and whose efficacy decays in time due to the emergence of fungicide resistant strains (Thomidis et al. 2009; Zhu et al. 2011).

Mathematical models, parametrized using appropriate data, offer great promise for the development of sustainable disease control strategies by allowing us to analyze the temporal dynamics of the disease and to predict the effect of different control strategies (Gilligan 2008). Considerable experimental evidence on brown rot in stone fruit, gathered in past years, identified correlations between the incidence (i.e., the proportion of fruit diseased out of the total number of observed fruit) of latent infection at early stages of fruit growth and the incidence of brown rot at harvest (Emery et al. 2000; Gell et al. 2008, 2009; Luo et al. 2005). Gibert et al. (2009) found that fruit cuticle cracks and the inoculum density played a major role in brown rot development. Yet, to our knowledge, there are no studies that have modeled the dynamic interactions between fruit exposure to the pathogen, fruit growth and cuticle cracking, and fruit infection to predict the trajectory of the disease progress.

†Corresponding author: D. Bevacqua; E-mail: daniele.bevacqua@inra.fr

Funding: This work has been partly carried out as part of the INRA Metaprogramme AAFCC (project CLIF) and the ECOVERGER project funded by ONEMA within the call APR "resistance et pesticides" managed by the MEDDE French Ministry.

*The $e$-Xtra logo stands for "electronic extra" and indicates that four supplementary figures and two supplementary tables are published online.

(C) 2018 The American Phytopathological Society
In the present work, we aim to fill this gap by developing and analyzing a model for the progression of brown rot in fruit orchards that captures these interactions. We derived the model, consisting of three epidemiological compartments and eight parameters, by modifying widely used epidemiological models (Gilligan 2008) in order to grasp the peculiarities of the brown rot pathosystem. We derived an intuitive expression for the basic reproduction number of the model that explicitly considers the fact that the latent period nonperiodically varies in time. We used an experimental peach (Prunus persica) orchard, which was not treated with fungicide and where environmental conditions allowed brown rot development, as a case study to set initial system conditions and estimate the model parameters. We compared the trajectories of disease progression predicted by the model with field observations. We performed a sensitivity analysis to investigate the response of the yield to the variation in the parameter estimates. We evaluated the effect of two common agricultural practices (namely, the winter elimination of blighted twigs and mummified fruit from the previous season to reduce the sources of primary inoculum and the control of the fruit load [FL] to obtain bigger fruit) on the yield by simulating their effect on the disease progression via their possible effect on the system initial conditions and on the model parameters.

\section{MATERIALS AND METHODS}

Brown rot infection in stone fruit orchards. We summarize below the essential features of the life cycle of Monilinia spp. in relation to brown rot disease progress in growing fruit. More detailed explanations can be found in previous dedicated studies (Martini and Mari 2014; Oliveira Lino et al. 2016; Rungjindamai et al. 2014). In the spring, in the presence of favorable conditions (mild temperatures and high relative humidity), mycelia and conidia of the fungus, which overwintered in mummified twigs and fruit, generate the so-called primary inoculum. The inoculum is composed of spores that are dispersed in the air by wind and rain and can be deposited on growing fruit, which we classify as being "exposed" to the pathogen. If this occurs in the first fruit growth 
stage, when the fruit is photosynthetically active and open stomata offer an entrance to the pathogen, the inoculum can penetrate into the fruit pulp, infect the fruit, reproduce, and generate secondary inoculum (Mari et al. 2003). If it occurs during the following pit-hardening stage, when the fruit is highly resistant to inoculum penetration, the spores can persist on the fruit cuticle until the fruit enters the third growth stage, when the cuticle cracks and pathogen penetration is facilitated. Once the pathogen penetrates the fruit and succeeds in reproduction, the fruit becomes infectious and generates secondary inoculum.

The model. Model outline and assumptions. The model describes the dynamics of the host fruit in a given orchard, from the end of the pit hardening growth stage, at time $t_{0}$, to harvest time, $t_{H}$, in relation to brown rot infection with growing fruit that can be susceptible $(S)$, exposed to the pathogen $(E)$, or infected and infectious $(I)$ (Fig. 1). The model is based on the following assumptions. The probability per time unit that a susceptible fruit is exposed to the pathogen is proportional to the density of infectious fruit. Exposed fruit return to the susceptible state at a constant rate, because the viability of the spores deposited on the fruit surface diminishes over time (Xu et al. 2001a), or they can progress to the infectious state at a rate that is proportional to the cuticle crack surface area (Gibert et al. 2009), which itself varies in time. Cuticle cracks start appearing when the fruit surpasses a critical size; thereafter, the cuticle crack surface area is proportional to the fruit mass (Gibert et al. 2007), which varies in time according to a logistic equation (Thornley and Johnson 1990). Infectious fruit are removed at a constant rate, because infectious fruit that dry out or absciss stop being sources of new inoculum (Oliveira Lino et al. 2016). In the absence of spatial autocorrelation in the incidence of infected fruit per tree, one can assume spatial homogeneity in the orchard.

Model equations. According to the model outline and assumptions, we can describe the dynamics of the epidemics, from $t_{0}$ to $t_{H}$, with the following system of ordinary differential equations (Fig. 1).

$$
\left\{\begin{array}{l}
\frac{d S}{d t}=\eta E-\lambda I S \\
\frac{d E}{d t}=\lambda I S-\eta E-\sigma(t) E \\
\frac{d I}{d t}=\sigma(t) E-\rho I
\end{array}\right.
$$

where $t$ represents the time, $\eta$ represents the rate at which the spores deposited on the fruit surface die or are wiped out and the fruit returns to the susceptible state (i.e., it is the inverse of the mean duration of spore viability on the fruit surface), $\lambda$ represents the per infectious unit rate at which susceptible individuals are exposed to the pathogen, $\sigma(t)$ represents the rate at which a fruit exposed to the pathogen transfers into the infectious compartment at time $t$, and $\rho$ represents the removal rate of an infectious fruit (i.e., it is the inverse of the mean duration of the infectious period). All the model variables are referred to fruit unit per $1 \mathrm{~m}^{2}$ of orchard surface $\left(\mathrm{U}_{\mathrm{F}}\right)$.

According to (Gibert et al. 2007), we set

$$
\sigma(t)=\left\{\begin{array}{lll}
0 & \text { if } & W(t)<w_{C} \\
\gamma W(t) & \text { if } & W(t)>w_{C}
\end{array}\right.
$$

where $\gamma$ is an infection constant, $w_{C}$ is the fruit weight threshold for cuticle cracking, and $W(\mathrm{t})$ is the fruit fresh weight at time $t$ during the growing season, which is calculated as

$$
W(t)=\frac{w_{B} w_{M}}{w_{B}+\left(w_{M}-w_{B}\right) e^{-k\left(t-t_{B}\right)}}
$$

where $t_{B}$ indicates the time at bloom; $w_{B}$ is the fruit weight at bloom; $w_{M}$ is the maximum fruit weight, which depends on the plant genotype and resources (e.g., carbon assimilates) availability; and $k$ is the conversion rate of resources into fruit mass (Thornley and Johnson 1990). After some algebra, we find that

$$
W(t)>w_{C} \text { when } t>\frac{1}{k} \log \frac{w_{C}\left(w_{M}-w_{B}\right)}{w_{B}\left(w_{M}-w_{C}\right)}+t_{B}
$$

A description of the model parameters and their units is summarized in Table 1.

The basic reproduction number, $\mathfrak{R}_{0}$, which represents the average number of secondary infection cases generated by a single primary infection in a completely susceptible population, is a key metric in epidemiology to understand the temporal spread of infections. Specifically, values of $\mathfrak{R}_{0}>1$ indicate that an infection will spread in a susceptible population, whereas values of $\mathfrak{R}_{0}<1$ indicates that an infection will decline. Although the expressions for $\mathfrak{R}_{0}$ have been rigorously defined in epidemic models with constant parameters (Diekmann and Heesterbeek 2000; van den Driessche and Watmough 2002) and periodically forced parameters (Bacaër and Guernaoui 2006), a rigorous expression for $\mathfrak{R}_{0}$ in models with parameters nonperiodically varying in time, such as model 1 , has not yet been derived. Here, we provide an intuitive expression to derive the basic reproduction number in model 1 , defined as $\mathfrak{R}_{0}\left(t_{l}, t_{H}\right)$, as a function of the time of primary infection $\left(t_{I}\right)$ into a host population that will be harvested at time $t_{H}$. In particular, we will define the number of secondary infections at time $t_{I}<t<t_{H}$ as the product of three terms: (i) the probability that a primary infectious individual introduced at time

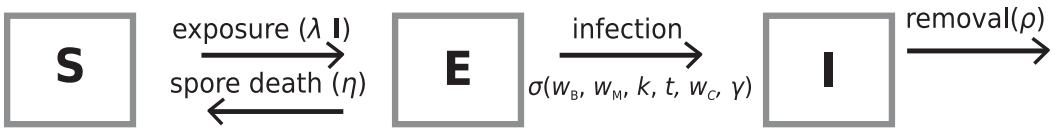

\begin{tabular}{|c|c|c|c|c|}
\hline Parameter & Definition & Value & $95 \%$ CI & Dimension \\
\hline$w_{B}$ & Fresh fruit weight at bloom time & 0.17 & {$[0.12 ; 0.21]$} & $\mathrm{g}$ \\
\hline$w_{M}$ & Maximum fresh fruit weight & 201 & {$[193 ; 210]$} & $\mathrm{g}$ \\
\hline$k$ & Conversion rate of resources into fruit mass & $6.7 \times 10^{-2}$ & {$[6.4 ; 7.1] \times 10^{-2}$} & day $^{-1}$ \\
\hline$\eta$ & Death rate of airborne spore & 0.13 & {$[0.004 ; 0.468]$} & day $^{-1}$ \\
\hline$\lambda$ & $\begin{array}{l}\text { Exposition rate of susceptible fruit per } \\
\text { infectious fruit }\end{array}$ & $5.8 \times 10^{-2}$ & {$[3.9 ; 37.0] \times 10^{-2}$} & $\mathrm{U}_{\mathrm{F}}^{-1}$ day $^{-1}$ \\
\hline ע & Infection constant & $1.5 \times 10^{-3}$ & {$[0.4 ; 2.4] \times 10^{-3}$} & $\mathrm{~g}^{-1} \mathrm{day}^{-1}$ \\
\hline
\end{tabular}

Fig. 1. Schematic diagram of the brown rot model with host fruit units that can be susceptible $(S)$, exposed $(E)$, or infectious $(I)$. See the text for details.

TABLE 1. Model parameters, definitions, estimated values (Value), and 95\% confidence intervals (CI) obtained by nonparametric randomization procedures and dimensions

${ }^{a}$ Dimensions are grams of fresh fruit mass $(\mathrm{g})$, days, and fruit unit per $1 \mathrm{~m}^{2}$ of orchard surface $\left(\mathrm{U}_{\mathrm{F}}\right)$. 
$t_{I}$ is still infectious at time $t$, (ii) the number of fruit that become exposed at time $t$, and (iii) the proportion of these exposed fruit that become infectious before $t_{H}$. Then, we define $\Re_{0}\left(t_{I}, t_{H}\right)$ as the integral of the number of secondary infections between $t_{I}$ and $t_{H}$. From our definition it follows that if $\mathfrak{R}_{0}\left(t_{I}, t_{H}\right)$ is $<1$ for every $t_{0}<t_{I}<t_{H}$, then an introduced infection will decline; whereas, if there exist some values of $t_{I}$ where $\Re_{0}\left(t_{I}, t_{H}\right)>1$, then there exist temporal windows where the infection can spread in the host population.

Model application to the peach-brown rot system. Available data. We collected data in 2014 from an experimental orchard of 43 peach trees (cultivar Magic/GF677) planted in 2006 at the INRA station of Avignon (southern France, $43^{\circ} 60 \mathrm{~N}, 4^{\circ} 49 \mathrm{E}$ ) initially composed of nine rows of five trees with 4-m spacing between trees. In the absence of fungicide treatment after bloom, brown rot naturally built up during spring and summer 2014, with no evidence of spatial autocorrelation in the incidence of infected fruit per tree (Supplementary Fig. S1). Apart from fungicide treatment, we managed trees according to conventional practices regarding fertilization, irrigation, and pesticide treatments. In mid-May, we carried out a light fruit thinning by removing, on average, $18 \%$ of fruit per shoot, thereby reducing the FL to 0.82 . Note that FL can vary from 0 to 1 , indicating the extreme cases in which no fruit is left over the tree and those in which no fruit is removed, respectively. From the beginning of June until harvest time in mid-July, we measured the abundance of symptomless and infectious fruit every week on 18 trees in the orchard after having excluded those on the border sides and a specimen that displayed low vigor. Hereinafter, with the term "symptomless", we refer to a fruit that does not present brown rot symptoms following a visual inspection in situ. Such a fruit can belong to the $S$ or $E$ compartments of the model. From the beginning of May until mid-July, we sampled 633 fruit at 15 different dates from the guard trees on the border sides to measure fruit fresh weight variation along the growing season (data are reported in Supplementary Table $\mathrm{S} 1$ ). Bloom time $\left(t_{B}\right)$ and harvest time $\left(t_{H}\right)$ occurred on 28 February and 15 July, respectively.

Parameter estimation. We estimated parameters $w_{B}, w_{M}$, and $k$ of the logistic equation (equation 3 ) by minimizing the mean squared error between the observed and predicted fruit weight at a given date. We obtained their empirical probability distributions by bootstrapping of the original data with 1,000 replicates (Efron and Tibshirani 1994). We set the value of the parameter $w_{C}=61 \mathrm{~g}$, according to Gibert et al. (2007), and we obtained its empirical probability distributions via 1,000 expectations computed as $y_{i}=\bar{y}+1.96 \epsilon_{i}$, where $\bar{y}$ is the average value and $\epsilon_{1}$ is a random sample from a normal distribution with mean 0 and standard deviation $=2.4 \mathrm{~g}$. We set the value of the parameter $\eta$ as the average of 12 estimates of airborne spore death rate computed from the data on airborne conidia viability in 12 different conditions reported by Xu et al. (2001a) for M. fructicola (Supplementary Table S2). We obtained an empirical probability distribution of $\eta$ via 1,000 random samples from the 12 different estimates. We estimated the values of the remaining unknown parameters and $\rho$ by minimizing the following cost function, describing the fraction of variances unexplained (FVU) by the model summed over different observable state variables,

$$
J(\lambda, \gamma, \rho)=\mathrm{FVU}_{S+E}(\lambda, \gamma, \rho)+\mathrm{FVU}_{1}(\lambda, \gamma, \rho)
$$

where

$$
\operatorname{FVU}_{i}(\lambda, \gamma, \rho)=\frac{\sum_{t}\left(\bar{y}_{i, t}-\hat{y}_{i, t}(\lambda, \gamma, \rho)\right)^{2}}{\sum_{t}\left(\bar{y}_{i, t}-\overline{\bar{y}}_{i}\right)^{2}}
$$

with $\bar{y}_{i, t}$ (with $i=S+E, I$ ) representing the observed density of symptomless (i.e., $S+E$ ) and infectious (i.e., $I$ ) fruit at sampling date $t, \hat{y}_{i, t}$ representing the corresponding estimate obtained from the model with a given parameter set, and $\overline{\bar{y}}_{i}$ representing the result of averaging $\bar{y}_{i, t}$ over all sampling dates. The advantage of using
FVU instead of mean squared errors is that FVU are comparable independently of the magnitude of the errors for different variables. A similar cost function was used in previous studies on biological control and fruit growth (Bruchou et al. 1999; Gubbins and Gilligan 1997). We assessed the empirical probability distributions for parameters $c, \lambda$, and $\rho$ by making use of (i) the empirical distributions of the five model parameters previously assessed and (ii) the moving block bootstrap, recommended for time series data (Kreiss and Lahiri 2012), to reconstruct time series of the observed variables (i.e., the density of symptomless and infectious fruit). Namely, (i) we drew a triad of parameters $\left(w_{B}, w_{M}, k\right)$ describing a fruit growth curve from the 1,000 replicates, a value of $w_{C}$ and one of $\eta$ from their empirical probability distributions; (ii) we reconstructed bootstrapped time series for each of the observed variable; and (iii) we assessed the values of parameters and $\rho$ by minimizing the cost function described in equation 5. We repeated this process 1,000 times. Minimization of the mean squared error between the observed and predicted fruit weight and of the cost function (equation 4) was performed using the Matlab function fminsearch (Nelder-Mead algorithm).

Model simulations and yield sensitivity to parameter estimate variation and agricultural practices. We numerically solved the model 1-2-3, simulating the infection dynamics from 3 June $\left(t_{0}\right), 95$ days after full bloom, to harvest time $\left(t_{H}\right)$, by using the MATLAB's ODE solver for ordinary differential equations ode45, which implements a Runge-Kutta method with a variable time step for efficient computation. We initially set $S\left(t_{0}\right)=8.94 \mathrm{U}_{\mathrm{F}}$, $E\left(t_{0}\right)=0.16$, and $I\left(t_{0}\right)=0.03 \mathrm{U}_{\mathrm{F}}$ according to field observations. Because field observations did not allow us to distinguish between susceptible and exposed fruit, we set $E\left(t_{0}\right)=1.8 \%$ of the entire fruit population according to Emery et al. (2000).

We used the model to simulate the system dynamics and we computed the yield as the fresh mass of symptomless, then marketable, fruit at harvest time. We examined the sensitivity of the yield to variation in model parameter estimates by varying each model parameter within the $95 \%$ confidence interval (CI) of its estimate (Table 1).We evaluated the consequences on the yield of sanitation practices, intended to control the abundance of primary inoculum, by adjusting the initial observed incidences of exposed and infectious fruit (i.e., 1.8 and $0.3 \%$ of the overall host population) with an initial infection factor (IF) varying from 0 to 1 , indicating the extreme cases in which no source of primary inoculum is present to start the infection and the case in which no sanitation takes place, respectively. Thus, we simulate the model 1-2-3 with the same initial overall host density (i.e., $9.13 \mathrm{U}_{\mathrm{F}}$ ) but with different proportions of $S, E$, and $I$.

We evaluated the consequences of fruit thinning practices by varying the FL between 0 and 1 . It is well known that the FL level affects the availability of resources for fruit growth and that bigger fruit are obtained at low FL (Gordon and Dejong 2007; Walcroft et al. 2004). Therefore, we both scaled the initial fruit density by the FL and adjusted the maximum fruit weight $\left(w_{M}\right)$ (which was originally estimated for FL $=0.82$ ) with a factor $\phi$. In the absence of any evidence on the shape of the negative relationship between $\phi$ and FL, we assumed $\phi=q-m F L$, where $q$ is the value of $\phi$ obtained in absence of competition between fruit and $m$ indicates the sensitivity of the fruit growth to FL. We assessed the values of $m=$ 1.24 and $q=2.02$ after some algebra by imposing that $\phi=1$ for $\mathrm{FL}=$ 0.82 , which corresponds to the value at which the fruit growth curve has been calibrated, and that final fruit size at extremely low FL (i.e., FL $\rightarrow 0$ ) is 2.6 times that obtained at extremely high FL (i.e., $\mathrm{FL}=1)($ Gibert et al. 2005).

\section{RESULTS}

Basic reproduction number, disease progress trajectories, and yield losses. The intuitive expression of the basic reproduction number $\mathfrak{R}_{0}\left(t_{l}, t_{H}\right)$ for model 1 , which represents the average number of secondary infectious cases generated by a single primary infection introduced at time $t_{I}$ (with $t_{0}<t_{I}<t_{H}$ ) in a 
completely susceptible population with a growing season ending at time $t_{H}$, is defined as follows:

$$
\mathfrak{R}_{0}\left(t_{I}, t_{H}\right)=\int_{t_{I}}^{t_{H}} e^{-\rho\left(t-t_{I}\right)} \lambda S\left(t_{I}\right)\left(\int_{t}^{t_{H}} \sigma(\tau) e^{-(\eta+\sigma(\tau))(\tau-t)} d \tau\right) d t
$$

Specifically, expression 6 is composed of three different terms representing the epidemiological processes involved in the disease transmission: (i) expression $e^{-\rho\left(t^{-} t_{I}\right)}$ represents the probability that a primary infectious fruit introduced at time $t_{I}$ is still infectious at time $t$; (ii) expression $\lambda S\left(t_{I}\right)$ represents the rate of exposure of susceptible individuals to conidia deriving from this infectious fruit, in a completely susceptible population (i.e., at the time of initial pathogen entry, $t_{I}$ ); and (iii) the integral

$$
\int_{t}^{t_{H}} \sigma(\tau) e^{-(\eta+\sigma(\tau))(\tau-t)} d \tau
$$

represents the probability of newly exposed individuals at time $t$ becoming infectious before time $t_{H}$, with $e^{-(\eta+\sigma(\tau))(\tau-t)}$ being the probability that an exposed fruit at time $t$ is still in the exposed class at time $\tau(>t)$, and $\sigma(\tau) d \tau$ being the probability of becoming infectious in the instant $d \tau$. In the case $\sigma(\tau)=\sigma$ (i.e., the exposure period is a constant and then time-independent) and $t_{H} \rightarrow+\infty$ (i.e., the fruit is not harvested), expression 6 becomes

$$
\mathfrak{R}_{0}\left(t_{I}, t_{H}\right)=\mathfrak{R}_{0}=\frac{\sigma \lambda S\left(t_{0}\right)}{(\eta+\sigma) \rho}
$$

which corresponds to the value of the basic reproduction number, $\mathfrak{R}_{0}$, obtained for epidemic models with constant parameters, as defined by Diekmann and Heesterbeek (2000).

Estimates of the model parameters and their CI are reported in Table 1. Fruit fresh weight increased from approximately $25 \mathrm{~g}$ to 150 to $225 \mathrm{~g}$ from May to mid-July, and its variation was well described by a logistic equation (Supplementary Fig. S2). Bootstrap distributions of the model parameters and their correlations, when they have been concurrently estimated, are reported in Supplementary Figures S3 and S4.
The reconstruction of the temporal dynamics of the disease progression and of the basic reproduction number, using average parameter values reported in Table 1, are shown in Figure 2. The density of exposed and infectious fruit remained very low until midJune, when the first infectious fruit permit inoculum reproduction and foster secondary infections (Fig. 2A). By the end of June the abundance of infectious fruit overcomes that of exposed ones and, in the week preceding the harvest, the abundance of infectious fruit overcomes that of symptomless ones (Fig. 2A). At harvest time, almost all fruit are infectious and their abundance starts declining as the density of susceptible fruit decreases sufficiently for the abundance of new infections to fall below that of infectious fruit removals (Fig. 2A). The model precisely fits the observed data at the outbreak of the epidemics and it overestimates the number of symptomless fruit only at harvest time (Fig. 2A). The values of $\Re_{0}\left(t_{I}, t_{H}\right)$ as a function of the time of primary introduction $t_{I}$ are shown in Figure 2B. The threshold for epidemic development, $\mathfrak{R}_{0}\left(t_{I}, t_{H}\right)=1$, is exceeded from the beginning of June, when model simulations start, until a few days before harvest, and $\mathfrak{R}_{0}\left(t_{I}, t_{H}\right)$ reached its maximum on 12 June.

When the model parameters are fixed to their average estimated value, average fruit fresh weight is equal to $180 \mathrm{~g}$ at the time of harvest (equation 3, when $t=196$ days, corresponding to 15 July). In the absence of the disease, the expected yield would be $1,644 \mathrm{~g} \mathrm{~m}^{-2}$ (approximately $26 \mathrm{~kg}$ tree $^{-1}$ ). In the presence of the disease, it is reduced to $276 \mathrm{~g} \mathrm{~m}^{-2}\left(4.4 \mathrm{~kg}\right.$ tree $\left.{ }^{-1}\right)$, with $47 \%$ of symptomless fruit nevertheless exposed to the pathogen.

Sensitivity of the yield to variation in the parameter estimate. The sensitivity of the yield to variation in the parameter estimate, in the presence of disease, is shown in Figure 3. An increase of the parameters $w_{B}, w_{M}$, and $k$, all corresponding to an increase in fruit growth (see equation 3 ), results in an increase of the infection rate, $\sigma(t)$, via its effect on the fruit size and on the cuticle crack surface. This leads to an increase in the brown rot incidence and a subtle decrease in the yield despite the increase in the fruit size. Varying the critical fruit size $\left(w_{C}\right)$ from 52 to $66 \mathrm{~g}$ has no consequences on the yield because, 2 days after the beginning of the simulations, the fruit mass already attained $66 \mathrm{~g}$ and cuticle cracks would appear whatever the value of $w_{C}$. Values of $w_{C}>66 \mathrm{~g}$ have a subtle positive effect on yield because they delay the time at which cracks occur and infection is made possible. An increase in the airborne spore death rate, corresponding to a decrease in the
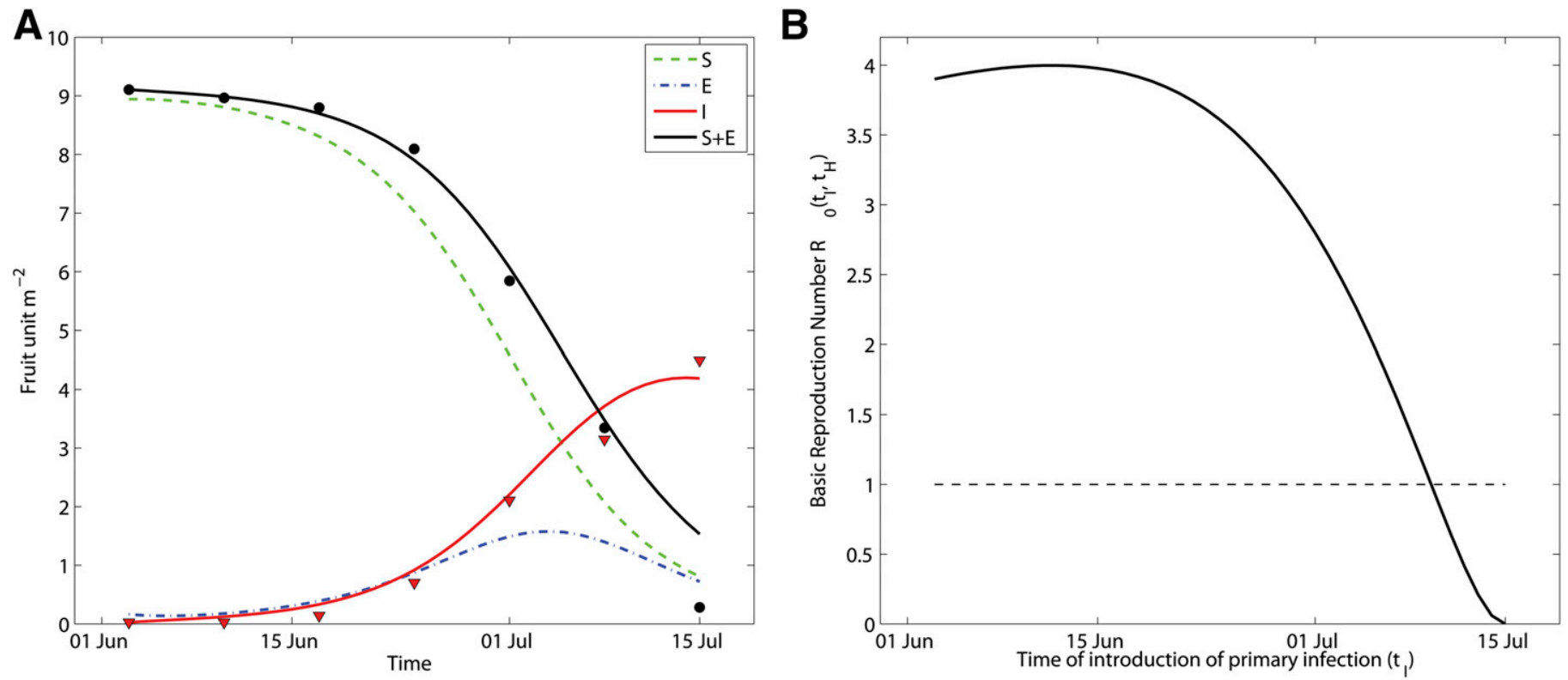

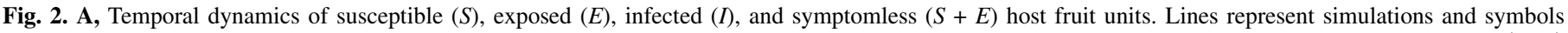

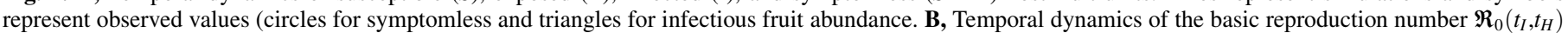

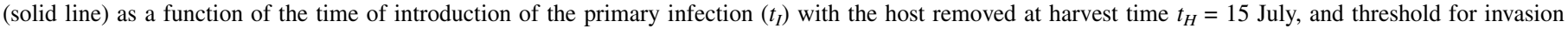
(dotted line). 
duration of spore viability, would increase the yield and severely decrease the proportion of the yield exposed to the pathogen. An airborne spore viability of only 2 days $(\eta=0.5)$ would allow a yield $>1,200 \mathrm{~g} \mathrm{~m}^{-2}$. Also, an increase in the exposition rate of susceptible fruit per infectious fruit $(\lambda)$ had a large impact on the epidemic, with the expected yield decreasing from 800 to $40 \mathrm{~g} \mathrm{~m}^{-2}$ when $\lambda$ increased from 0.04 to $0.10 \mathrm{U}_{\mathrm{F}}^{-1}$ day $^{-1}$. Similarly, an increase in the infection constant $(\gamma)$ also would determine an increase in the disease incidence and important yield losses. Yet, a decrease of the infection constant would determine a yield of approximately $1,500 \mathrm{~g} \mathrm{~m}^{-2}$. Even a higher yield would be obtained for an infectious period of $\rho^{-1}$ lower than 3 days.

Sensitivity of the yield to agricultural practices. The response of the yield, in terms of quantity and composition, to different values of IF and FL is shown in Figure 4. In the impossibility of disease spreading, the initial incidence of infection does not progress and the yield would be slightly $>1,600 \mathrm{~g} \mathrm{~m}^{-2}$ but, when disease spreading is possible, small initial infection incidences (i.e., 20\% of the incidence observed in the field in 2014) are responsible for a $50 \%$ reduction of the yield (Fig. 4A). Moreover, at these initial values of infection density, almost one-third of the yield was composed of fruit exposed to the pathogen (Fig. 4A). The response of the yield to different FL values was particularly interesting (Fig. 4B). The effect of FL on yield was nonmonotonic, with initial increases in yield for higher FL up to an FL of approximately 0.88 (in the impossibility of disease spreading), at which point lower fruit size resulted in a decrease in yield. In the presence of disease spreading, this inflection point was reached much earlier, at an FL of 0.36. In absolute terms, when disease spread is possible, the maximum yield of symptomless fruit $\left(786 \mathrm{~g} \mathrm{~m}^{-2}\right)$ is obtained at $\mathrm{FL}=$ 0.36 . However, it is worth noting that such a yield would contain a significant fraction (approximately 9\%) of fruit exposed to the pathogen and that average fruit size would be $284 \mathrm{~g}$. On the other hand, a slightly lower value of FL (approximately 0.25 ) would allow for an almost brown-rot-free yield ( $<1 \%$ of exposed fruit) of $697 \mathrm{~g} \mathrm{~m}^{-2}$ and an average fruit size of $307 \mathrm{~g}$.

\section{DISCUSSION}

In this work, we developed the first epidemic model for brown rot progression in fruit orchards by modifying classical compartmental epidemiological susceptible, exposed, infectious, and removed models (Gilligan 2008; Madden et al. 2007). Specifically, we (i) added the possibility to return from the $E$ to the $S$ class because the viability of the spores deposited on the fruit surface is time limited; (ii) assumed the probability to pass from the $E$ to the $I$ class is dependent on the state of the fruit, which itself varies in time, because the exposure to the pathogen is not expected to result in infectivity if the fruit cuticle is entire; and (iii) removed the recovered (immune) class, because the fruit is unable to recover once the pathogen accesses the pulp and succeeds in reproducing. The proposed framework, specifically developed for brown rot infection in stone fruit, could be applied to different fruit crops and plant diseases exhibiting the same peculiarities. For instance, in the grapevine-Botrytis cinerea system, the fungal development is inhibited until berry ripening (Deytieux-Belleau et al. 2009; Kretschmer et al. 2007). Also, in the apple and pear-M. fructigena system, the epidemic spreads when the fruit skin is damaged by insects or birds (Gell et al. 2009; Xu et al. 2001b).

Basic reproduction number, disease progress trajectories, and yield losses. By assuming a time variant latency period of $\sigma(t)^{-1}$, we obtained a time variant intuitive expression for the basic reproduction number $\mathfrak{R}_{0}\left(t_{I}, t_{H}\right)$ that can be used to evaluate the risk of epidemic spread for infections introduced at different times in the fruit growing season and, consequently, to optimize the timing and strength of treatments (e.g., fungicide application). The fact that $\mathfrak{R}_{0}\left(t_{I}, t_{H}\right)$ was above the critical threshold for invasion until 10 July indicates that actual environmental conditions met in 2014 were very favorable to the disease, and also suggests that treatments should have been maximum in mid-June. This result suggests that fruit treatments would be more effective in the first part of the summer than closer to harvest time. This is particularly valuable because treatments applied just before harvest are the most harmful to human health (Lippmann and Leikauf 2008).
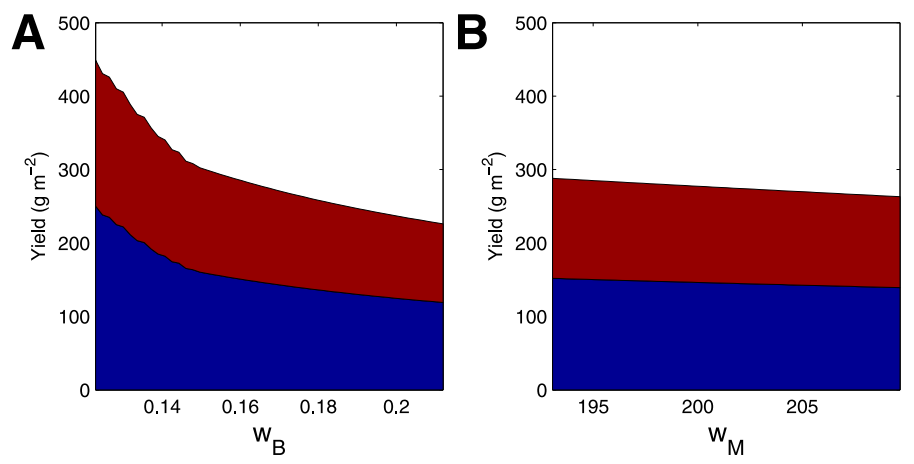

$\mathbf{E}$

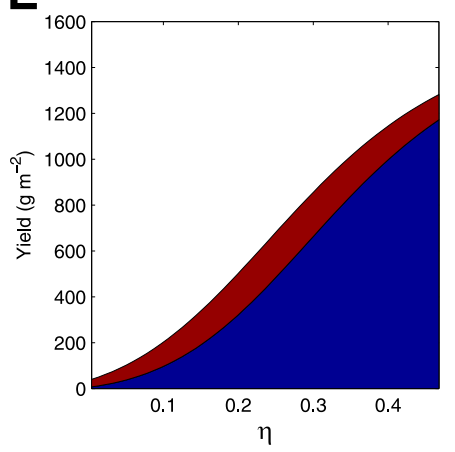

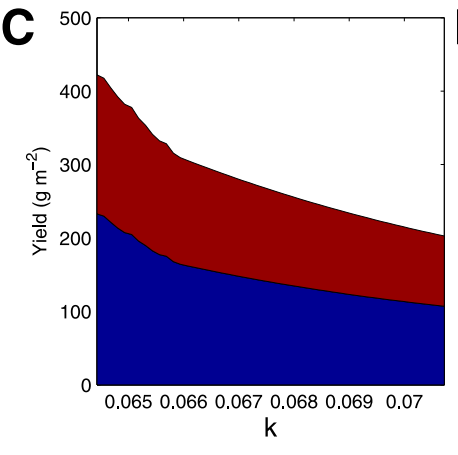

G

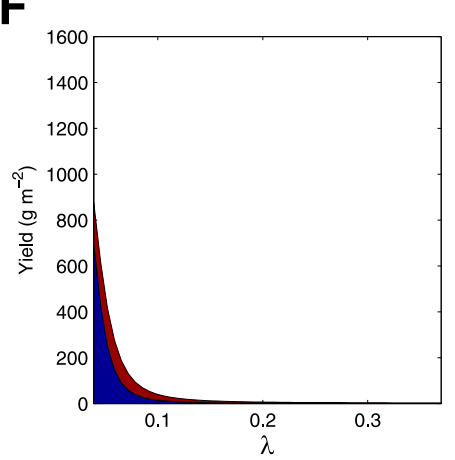

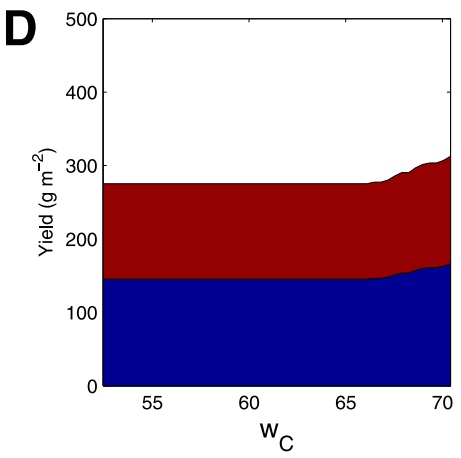

H

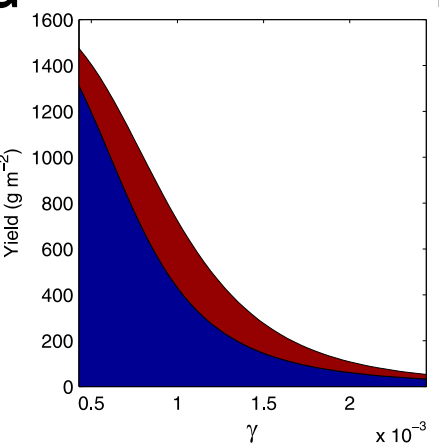

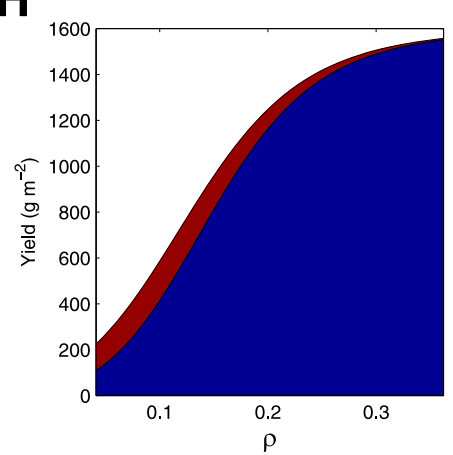

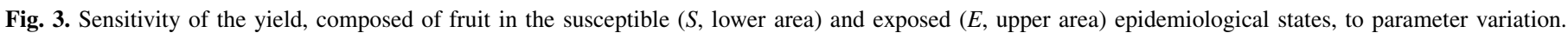

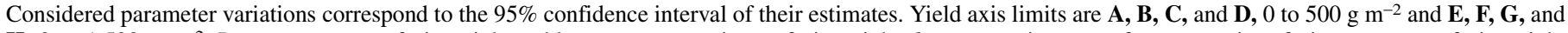

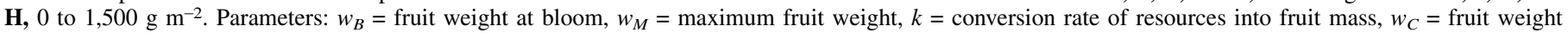

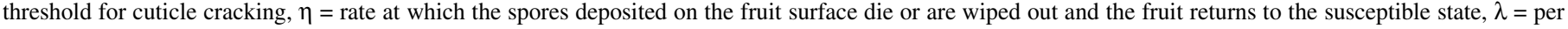
infectious unit rate at which susceptible individuals are exposed to the pathogen, $\gamma$ is an infection constant, and $\rho=$ the removal rate of infectious fruit. 
The fact that environmental conditions met in 2014 were optimal for brown rot build up are confirmed by the observed disease incidence at harvest (i.e., $94 \%$ of rotten fruit), which exceeds values observed in previous studies on peach (Emery et al. 2000; Gell et al. 2008; Gibert et al. 2009; Keske et al. 2011). Our model closely reproduces the observed temporal dynamics of the peach-brown rot pathosystem. The density of infectious fruit remained low until mid-June and increased until harvest time, with a maximum increase 12 days before harvest. Similarly, previous field studies found low brown rot incidence until 7 to 12 days before harvest in peach cultivars in Georgia (United States) and Spain (Emery et al. 2000; Gell et al. 2009). At the very end of the study period, the model overestimated the number of symptomless fruit. This might be due to the fact that, in the last phase of the growing season, fruit density may reduce due to natural fruit abscission (Estornell et al. 2013).

Sensitivity of the yield to variation in the parameter estimate. The results of the sensitivity analysis depend on the tested parameter space. In the present work, such a space reflects the variation in the parameter estimates, which were based on our experimental field data. Such data exhibited a very high disease incidence at harvest time and, consistently, the model predicts important yield losses all over the tested parameter space, with exceptions for the lowest values of the average airborne spore viability $\left(\eta^{-1}\right)$, the infection constant $(\gamma)$, and the average infectious period $\left(\rho^{-1}\right)$. Because it is well acknowledged that brown rot epidemics do not occur under unfavorable environmental conditions (Gell et al. 2008; Koball et al. 1997; Xu et al. 2001a), we expect that the model parameters display values at the border or even outside of the tested space in different environmental conditions.

Nevertheless, the performed sensitivity analysis allowed us to assess the robustness of the model predictions and provided an insight into the functioning of the system to better comprehend the role possibly played by environmental factors and agricultural practices. Our analysis indicates that a decrease in the fruit growth rate, which is commonly undesirable to growers because it would reduce fruit size, increases the yield by decreasing brown rot incidence. These results are in agreement with the empirical observation of Mercier et al. (2008), who found that water deprivation resulted in both lower fruit size and lower brown rot incidence. A longer spore viability $\left(\eta^{-1}\right)$ determines a lower yield because it increases the probability of persistence until the fruit cuticle starts cracking. The suggestion that "latent infections were most

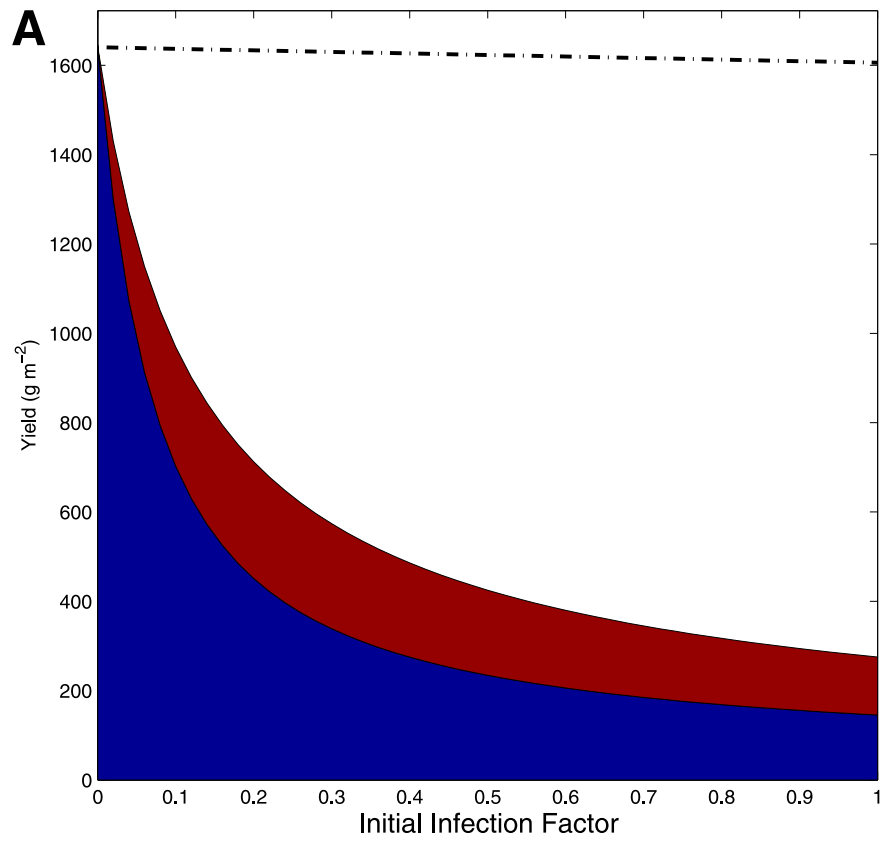

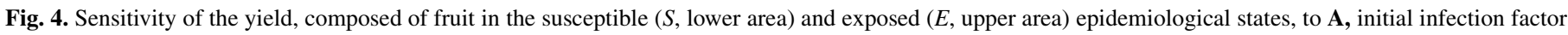

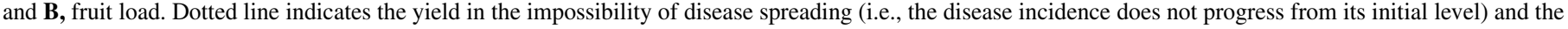
white area below the dotted line indicates the yield loss due to the brown rot.

important in humid temperate regions" (Emery et al. 2000) may be due to a lower value of $\eta^{-1}$ in those years with lower relative humidity (Xu et al. 2001a). As expected, the yield is very sensitive to the variation in the estimate of the exposure rate $(\lambda)$. This parameter depends on the spore production by infectious fruit, their dispersal in the orchard, and the probability that they land on the fruit surface, which might increase with the fruit size. Both spore production and dispersal are influenced by meteorological conditions, with optimal production of $M$. fructicola in peach observed at $15^{\circ} \mathrm{C}$ (Phillips 1984) and dispersal favored by wind and rain (Byrde and Willetts 1977). Systemic fungicides that reduce spore production are expected to reduce the parameter $\lambda$. The infection constant $(\gamma)$ governing the progress from the exposed to the infectious state also plays a major role in brown rot epidemics. This parameter is affected by environmental conditions, physical and chemical barriers of the fruit, and the possible application of protectant fungicides (Oliveira Lino et al. 2016). Fourie and Holz (2003) found that plum fruit developed brown rot disease only after prolonged periods $(>12 \mathrm{~h})$ of wet incubation. Other authors found that a temperature of 23 to $25^{\circ} \mathrm{C}$ and relative humidity $>97 \%$ was optimal for $M$. fructigena development in apple and pear (Luo et al. 2001; Xu et al. 2001a). Novel breeding schemes intended to design genotypes less inclined to cuticle cracking (Quilot-Turion et al. 2012) could provide crop varieties with lower values for this parameter. Fungicide application is also expected to reduce $\gamma$. Finally, we found that the variation in the estimate of the average infectious period $\left(\rho^{-1}\right)$ has the highest impact on the yield. Our results suggest that practices intended to remove infectious fruit during the growing season could significantly help in controlling brown rot by decreasing the average infectious period. Even in the presence of favorable conditions for brown rot build up, if the infectious fruit were removed every 4 days, brown rot could be efficiently controlled.

Sensitivity of the yield to agricultural practices. We found that the yield exponentially decrease with the initial infection level (Fig. 4A). Similar results were observed in previous field studies on the relationship between disease incidence at harvest and early infection (Emery et al. 2000; Villarino et al. 2010) and indicate that winter sanitation practices are efficient only when capable of removing almost all the sources of primary inoculum; otherwise, they would not produce significant results.

The unimodal relationship between yield and FL (Fig. 4B) results from higher densities of fruit at higher FL (which facilitate exposure to spores) but lower values of cuticle cracking (which reduces the

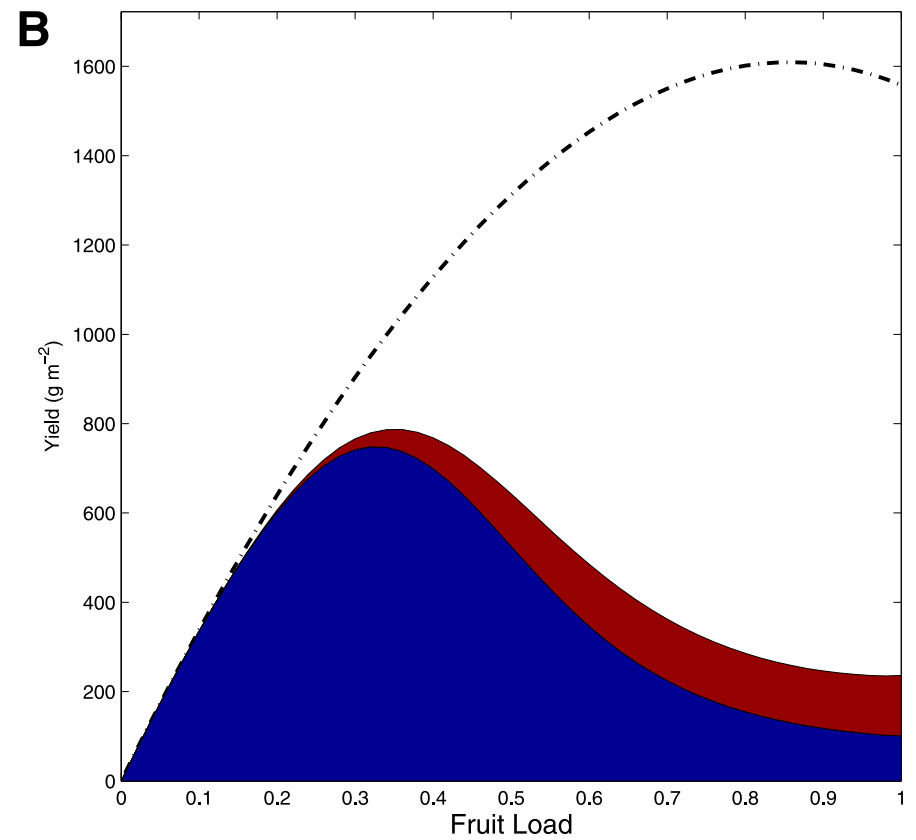


rate of infection of exposed fruit). Gibert et al. (2007) observed this second mechanism; they found that fruit grown on trees at higher FL had a lower probability of becoming infectious after artificial inoculation with Monilinia spp. conidia. Such a result alone may lead one to believe that high FL can reduce brown rot spreading in the field. On the other hand, our epidemiological approach suggests that an intermediate level of FL would maximize the yield in the presence of disease and highlights the importance of evaluating the consequences of agricultural practices on disease control in a comprehensive epidemiological framework.

Finally, our work points out that agricultural practices have implications not only for yield quantity but also for quality because they can affect both the fruit size and the proportion of the yield that is exposed to the pathogen and, therefore, at increased risk of rotting in the postharvest phase. Although postharvest fruit rotting is a major problem in the fruit market (Larena et al. 2005; Rungjindamai et al. 2014) and undersized fruit have no economic value (Grechi et al. 2012), the qualitative composition of the yield is often neglected in crop pathology; therefore, we are convinced that the proposed modeling framework could assist in the development of orchard management strategies considering the quality in addition to the quantity of the yield.

\section{ACKNOWLEDGMENTS}

We thank M. Gènard and F. Lescourret for fruitful discussions, A. Tran and V. Signoret for the field work, and two anonymous referees for extremely valuable comments.

\section{LITERATURE CITED}

Bacaër, N., and Guernaoui, S. 2006. The epidemic threshold of vector-borne diseases with seasonality: The case of cutaneous leishmaniasis in Chichaoua, Morocco. J. Math. Biol. 53:421-436.

Bruchou, C., Génard, M., and Génard, M. 1999. A space-time model of carbon translocation along a shoot bearing fruits. Ann. Bot. (Lond.) 84:565-576.

Byrde, R., and Willetts, H. 1977. The Brown Rot Fungi of Fruit: Their Biology and Control. Pergamon Press, Oxford, UK.

Deytieux-Belleau, C., Geny, L., Roudet, J., Mayet, V., Donèche, B., and Fermaud, M. 2009. Grape berry skin features related to ontogenic resistance to Botrytis cinerea. Eur. J. Plant Pathol. 125:551-563.

Diekmann, O., and Heesterbeek, J. 2000. Mathematical Epidemiology of Infectious Diseases: Model Building, Analysis and Interpretation. John Wiley \& Sons, New York

Efron, B., and Tibshirani, R. J. 1994. An Introduction to the Bootstrap, 1st ed. Monographs on Statistics \& Applied Probability 57. Chapman and Hall/CRC, London, UK.

Emery, K. M., Michailides, T. J., and Scherm, H. 2000. Incidence of latent infection of immature peach fruit by Monilinia fructicola and relationship to brown rot in Georgia. Plant Dis. 84:853-857.

Estornell, L. H., Agustí, J., Merelo, P., Talón, M., and Tadeo, F. R. 2013. Elucidating mechanisms underlying organ abscission. Plant Sci. 199-200:48-60.

Fourie, P. H., and Holz, G. 2003. Germination of dry, airborne conidia of Monilinia laxa and disease expression on nectarine fruit. Australas. Plant Pathol. 32:9-18.

Gell, I., De Cal, A., Torres, R., Usall, J., and Melgarejo, P. 2008. Relationship between the incidence of latent infections caused by Monilinia spp. and the incidence of brown rot of peach fruit: Factors affecting latent infection. Eur. J. Plant Pathol. 121:487-498.

Gell, I., De Cal, A., Torres, R., Usall, J., and Melgarejo, P. 2009. Conidial density of Monilinia spp. on peach fruit surfaces in relation to the incidences of latent infections and brown rot. Eur. J. Plant Pathol. 123:415-424.

Gibert, C., Chadoeuf, J., Nicot, P., Vercambre, G., Génard, M., and Lescourret, F. 2009. Modelling the effect of cuticular crack surface area and inoculum density on the probability of nectarine fruit infection by Monilinia laxa. Plant Pathol. 58:1021-1031.

Gibert, C., Chadœuf, J., Vercambre, G., Génard, M., and Lescourret, F. 2007. Cuticular cracking on nectarine fruit surface: Spatial distribution and development in relation to irrigation and thinning. J. Am. Soc. Hortic. Sci. 132:583-591.

Gibert, C., Lescourret, F., Génard, M., Vercambre, G., and Pérez Pastor, A. 2005. Modelling the effect of fruit growth on surface conductance to water vapour diffusion. Ann. Bot. (Lond.) 95:673-683.

Gilligan, C. 2008. Sustainable agriculture and plant diseases: An epidemiological perspective. Philos. Trans. R. Soc. Lond. B Biol. Sci. 363:741-759.

Gordon, D., and Dejong, T. M. 2007. Current-year and subsequent-year effects of crop-load manipulation and epicormic-shoot removal on distribution of long, short and epicormic shoot growth in Prunus persica. Ann. Bot. (Lond.) 99:323-332.

Grechi, I., Ould-Sidi, M., Hilgert, N., Senoussi, R., Sauphanor, B., and Lescourret, F. 2012. Designing integrated management scenarios using simulationbased and multi-objective optimization: Application to the peach tree-Myzus persicae aphid system. Ecol. Modell. 246:47-59.

Gubbins, S., and Gilligan, C. 1997. Biological control in a disturbed environment. Philos. Trans. R. Soc. Lond. B Biol. Sci. 352:1935-1949.

Keske, C., Amorim, L., and May-De Mio, L. 2011. Peach brown rot incidence related to pathogen infection at different stages of fruit development in an organic peach production system. Crop Prot. 30:802-806.

Koball, D., Wilcox, W., and Seem, R. 1997. Influence of incubation-period humidity on the development of brown rot blossom blight of sour cherry. Phytopathology 87:42-49.

Kreiss, J., and Lahiri, S. N. 2012. Bootstrap Methods for Time Series. Int. Stat. Inst. 30:1-26.

Kretschmer, M., Kassemeyer, H. H., and Hahn, M. 2007. Age-dependent grey mould susceptibility and tissue-specific defence gene activation of grapevine berry skins after infection by Botrytis cinerea. J. Phytopathol. 155: 258-263.

Larena, I., Torres, R., De Cal, A., Liñán, R., Melgarejo, P., Domenichini, P., Bellini, A., Mandrin, J. F., Lichou, J., Ochoa de Eribe, X., and Usall, J. 2005. Biological control of postharvest brown rot (Monilinia spp.) of peaches by field applications of Epicoccum nigrum. Biol. Control 32:305-310.

Lippmann, M., and Leikauf, G. 2008. Introduction and background. Pages 1-38 in: Environmental Toxicants: Human Exposures and Their Health Effects, 3rd ed. M. Lippmann, ed. Wiley, Hoboken, NJ.

Luo, Y., Michailides, T. J., Morgan, D. P., Krueger, W. H., and Buchner, R. P. 2005. Inoculum dynamics, fruit infection, and development of brown rot in prune orchards in California. Phytopathology 95:1132-1136.

Luo, Y., Morgan, D. P., and Michailides, T. J. 2001. Risk analysis of brown rot blossom blight of prune caused by Monilinia fructicola. Phytopathology 91: 759-768.

Madden, L., Hughes, G., and van den Bosch, F. 2007. The Study of Plant Disease Epidemics. The American Phytopathological Society, St. Paul, MN.

Mari, M., Casalini, L., Baraldi, E., Bertolini, P., and Pratella, G. C. 2003. Susceptibility of apricot and peach fruit to Monilinia laxa during phenological stages. Postharvest Biol. Technol. 30:105-109.

Martini, C., and Mari, M. 2014. Postharvest Decay. Pages 233-265 in: Monilinia fructicola, Monilinia laxa (Monilinia Rot, Brown Rot). S. Bautista-Banos, ed. Academic Press, San Diego, CA.

Mercier, V., Bussi, C., Plenet, D., and Lescourret, F. 2008. Effects of limiting irrigation and of manual pruning on brown rot incidence in peach. Crop Prot. 27:678-688

Oliveira Lino, L., Pacheco, I., Mercier, V., Faoro, F., Bassi, D., Bornard, I., and Quilot-Turion, B. 2016. Brown rot strikes Prunus fruit: An ancient fight almost always lost. J. Agric. Food Chem.

Phillips, D. 1984. Effect of temperature on Monilinia fructicola spores produced on fresh stone fruits. Plant Dis. 68:610-612.

Quilot-Turion, B., Ould-Sidi, M., Kadrani, A., Hilgert, N., Génard, M., and Lescourret, F. 2012. Optimization of parameters of the "Virtual Fruit" model to design peach genotype for sustainable production systems. Eur. J. Agron. 42:34-48.

Rungjindamai, N., Jeffries, P., and Xu, X. M. 2014. Epidemiology and management of brown rot on stone fruit caused by Monilinia laxa. Eur. J. Plant Pathol. 140:1-17.

Thomidis, T., Michailides, T., and Exadaktylou, E. 2009. Contribution of pathogens to peach fruit rot in northern Greece and their sensitivity to iprodione, carbendazim, thiophanate-methyl and tebuconazole fungicides. J. Phytopathol. 157:194-200.

Thornley, J., and Johnson, I. 1990. Plant and Crop Modeling: A Mathematical Approach to Plant and Crop Physiology. Clarendon Press, Oxford, UK.

van den Driessche, P., and Watmough, J. 2002. Reproduction numbers and sub-threshold endemic equilibria for compartmental models of disease transmission. Math. Biosci. 180:29-48.

Villarino, M., Melgarejo, P., Usall, J., Segarra, J., and De Cal, A. 2010. Primary Inoculum Sources of Monilinia spp. in Spanish Peach Orchards and their relative importance in brown rot. Plant Dis. 94:1048-1054.

Walcroft, A., Lescourret, F., Génard, M., Sinoquet, H., Le Roux, X., and Donès, N. 2004. Does variability in shoot carbon assimilation within the tree crown explain variability in peach fruit growth? Tree Physiol. 24:313-322.

Xu, X.-M., Guerin, L., and Robinson, J. D. 2001a. Effects of temperature and relative humidity on conidial germination and viability, colonization and sporulation of Monilinia fructigena. Plant Pathol. 50:561-568.

Xu, X.-M., Robinson, J. D., Berrie, A. M., and Harris, D. C. 2001b. Spatiotemporal dynamics of brown rot (Monilinia fructigena) on apple and pear. Plant Pathol. 50:569-578.

Zhu, X., Chen, X., and Guo, L. 2011. Population structure of brown rot fungi on stone fruits in China. Plant Dis. 95:1284-1291. 